\title{
Procesos curriculares desde la deconstrucción en la enseñanza de las ciencias
}

\section{Curricular processes since the deconstruction in the teaching of sciences}

\author{
Claudia Patricia Naranjo Zuluaga ${ }^{1}$ \\ Facultad de Educación y Ciencias Humanas, \\ Universidad de Córdoba, Colombia. Grupo de Investigación GICNEA \\ Julio Simón Garay Carrascal ${ }^{2}$ \\ Facultad de Educación y Ciencias Humanas, Universidad de Córdoba, Colombia
}

\section{RESUMEN}

El presente trabajo investigativo tiene como propósito fundamental la integralidad curricular para la enseñanza de las ciencias naturales, a partir de procesos de deconstrucción en el ejercicio docente. Para ello se pone en marcha el uso de un método de estudio de casos aplicado al docente de ciencias naturales del grado séptimo de la Institución Educativa Cristóbal Colon del Corregimiento Campanito Municipio

$1 \quad$ Magíster en Educación, Docente catedrática en la Licenciatura en Ciencias Naturales y Educación Ambiental, cpnaranjo@correo.unicordoba.edu.co, teléfono:3206740234, https://orcid.org/0000-0001-9209-9586

2 Magíster (c) en Didáctica de las Ciencias Naturales, juliogarayc@correo.unicordoba.edu.co. teléfono: 3159286533, https://orcid.org/0000-0001-7095-1846 Facultad de Educación y Ciencias Humanas, Universidad de Córdoba, Colombia. de Cereté, Córdoba, caracterizado por una fase investigativa que aborda tres momentos específicos: un primer momento de análisis conceptual de la estructura curricular propuesta en la Institución Educativa objeto de indagación, seguido de un segundo momento sobre la interpretación del ejercicio docente, mediante la aplicación de una entrevista semiestructurada, cuyo análisis estará correspondido con el uso de Atlas-ti. Finalmente, un tercer momento en la deconstrucción en el que se realiza un análisis documental al plan curricular de ciencias naturales del grado objeto de estudio, desde el enfoque de integralidad. A la luz de los instrumentos aplicados, se constatan resultados que conciben una práctica que deja manifiesto la necesidad de la construcción de una posible 
propuesta de currículo integral contextualizado que le permita al estudiante entender la realidad de su naturaleza y confiera respuestas a situaciones de su vida cotidiana.

PALABRAS CLAVE: Integralidad curricular, planeación, ejercicio docente, plan curricular.

\section{ABSTRACT}

The present investigative work has as its fundamental purpose the curricular integrality for the teaching of natural sciences, from deconstruction processes in the teaching exercise. For this, the use of a case study method applied to the seventh grade natural sciences teacher of the Cristóbal Colon Educational Institution of the Corregimiento Campanito Municipality of Cereté, Córdoba, characterized by an investigative phase that addresses three specific moments: a first moment of conceptual analysis of the curricular structure proposed in the Educational Institution under investigation, followed by a second moment on the interpretation of the teaching exercise, through the application of a semi-structured interview, the analysis of which will correspond to the use of Atlas-ti . Finally, a third moment in the deconstruction in which a documentary analysis is made of the natural sciences curriculum of the grade under study, from the integral approach. In the light of the applied instruments, results are found that conceive a practice that reveals the need for the construction of a possible proposal for a comprehensive contextualized curriculum that allows the student to understand the reality of their nature and confers responses to situations in their life everyday.

\section{KEYWORDS}

Curricular comprehensiveness, planning, teaching exercise, curricular plan

\section{INTRODUCCIÓN}

El currículo integrado es toda modalidad que permita organizar y planificar diversos temas pedagógicos con el propósito de lograr la integración ysocializaciónen laprácticaeducativa dirigida a fin de tomar decisiones competentes en un entorno cultural educativo (Jelves, 2017). La implementación de un currículo integrado permite al estudiante adquirir habilidades de aprendizaje a largo plazo, construyendo su propio proceso sobre lo ya aprendido de una manera flexible e individualizada (RodríguezLearte et al., 2018) genéticos y fisiológicos de los procesos biológicos, y ello contribuye a una mejor comprensión del funcionamiento normal de nuestro cuerpo y establece el conocimiento de las bases de la patología. Esto implica que los profesionales de ciencias de la salud deben desarrollar competencias y capacidades especiales que les permitan establecer nexos dinámicos entre las ciencias básicas y su práctica profesional. El dise- ño curricular más adecuado para la formación en estas competencias y capacidades se logra a través del currículo integra- do. El aprendizaje integrado es un proceso centrado en el alumno, mediante el cual se adquieren conocimientos de manera flexible e individualizada a largo plazo. En la Universidad Europea de Madrid hemos afrontado esta nueva necesi- dad utilizando un modelo de aprendizaje integrado de materias básicas indicado para abordar la integración curricular progresiva, y que hemos denominado WSLA (Work Stations Learning Activities.

Para esta investigación es determinante la aplicación de procesos curriculares hacia la complejidad, en contraposición con los enfoques transmisivos. De esta manera, el accionar docente, debe asumir un cambio substancial en la enseñanza de las ciencias naturales hacia la integralidad; de tal modo que se promuevan escenarios transformadores en constantes cambios, que trascienden en los 
problemas educativos, donde el docente debe articular esos cambios en su actuar, generando aprendizajes más flexibles, abiertos, asociados a la realidad contextual y la vida cotidiana del estudiante (Lapasta, L. 2018).

La visión para la enseñanza de las ciencias no se debe concebir de una manera aislada y fragmentaria, por el contrario, requiere una concepción de un todo, donde trascienda la disciplina y el estudiante sea protagonista de su propia formación, con una visión crítica, íntegra y emancipadora (Marín, 2012). De manera que, el docente es mediador, con intuición deliberadora, consciente de seleccionar estrategias que permitan la integración del proceso del aprender y su aplicación contextual (Marín, G., Paredes, C., e Inciarte, G. 2017).

Como consecuencia de un rastreo bibliográfico respecto a los temas de interés objeto de esta investigación, se encuentran algunos referentes que a partir de postulados teóricos relacionados con el currículo integral (Pinto, 2008, entre otros); la interdisciplinariedad (Morin, 2003; Schunk, 2012, entre otros) y relevancias teóricas de las ciencias naturales a partir de sus procesos de enseñanza - aprendizaje (López, R. 1990; Valbuena, 2007). La literatura científica desde contextos internacionales (Lozano, C. y Villanueva, G. 2016; entre otros); y nacionales (Hernández et al. 2018, entre otros autores); fundamentan y contrastan los resultados encontrados a la luz del análisis de los objetivos desarrollados, en correspondencia con las bases conceptuales, teoricidad y aspectos metodológicos pertinentes.

El objetivo del presente estudio, es interpretar los procesos curriculares a partir de la deconstrucción de la enseñanza de las ciencias naturales desde un análisis hermenéutico de la estructura curricular, del ejercicio de planeación docente y del plan curricular. En correspondencia teórica con el propósito que moviliza este trabajo investigativo, se señalan algunos aspectos relevantes de la significancia de la deconstrucción de procesos de enseñanza y aprendizaje entrándose en la obligatoriedad de comprender los aspectos teórico - conceptual y metodológico del mismo.

En ese orden de ideas, Aguilar, Bermúdez y Hernández (2018), especifican que la deconstrucción ha sido abordada desde la teoría y el pragmatismo, además ha sido llevada a los contextos de la práctica pedagógica a través de los postulados de Restrepo (2006), cuyo objetivo promueve una fase investigativa como "un proceso que trasciende más allá de un autoexamen de la práctica para entrar en diálogos más amplios con componentes que explican la razón de ser de las tensiones que la práctica enfrenta" (p. 96). Es decir desde una perspectiva reflexiva de los procesos de enseñanza - aprendizaje dentro del aula.

Ahora bien, para efectos de los procesos de deconstrucción de la enseñanza de las ciencias, el docente debe considerar sus aspectos epistemológicos: normativas, cumplimiento interdisciplinar, estándares y competencias, procesos metodológicos, quehacer fragmentado, aunque esto implique procesos de resignificación curricular a partir de una perspectiva integral o interdisciplinar como se recomienda en los estándares (Hernández, et al. 2018; Vivas, Mancipe y Aguilar, 2017).

\section{METOLOGÍA}

La metodología a partir de la cual se orienta esta investigación es la cualitativa, vista como el intento de obtener una comprensión profunda de los significados y definiciones de la situación tal como nos la presentan las personas, más que la producción de una medida cuantitativa de sus características o conducta (Salgado, 2007, p. 71). Al respecto, utiliza la recolección y análisis de los datos para afinar las preguntas de investigación o revelar nuevos interrogantes 
en el proceso de interpretación (Hernández, Fernandez, \& Baptista, p, 2014, p.7).

El aspecto metodológico que sustenta la investigación cualitativa caracteriza métodos y técnicas diversas como gama de estrategias que ayudarán a reunir los datos que van a emplearse para la inferencia y la interpretación, para la explicación y la predicción (Munarriz, 1992, p. 103). Sin embargo, antes de especificarse el tipo de investigación o método a utilizar en este trabajo investigativo, se acude a las consideraciones expuestas por Monje, C. (2011), quien aclara que cualquier método o tipo de investigación tiene un diseño específico que permite aplicar las técnicas e instrumentos específicos para resolver los interrogantes planteados en la investigación. Algunos tienen sus ventajas y desventajas o limitaciones en sus resultados, siendo unos más adecuados que otros.

Ahora bien, atendiendo al proceso de deconstrucción de la enseñanza y los elementos metodológicos aplicados para el abordaje de esta fase, se atiende un tipo de investigación o método de estudio de caso, el cual es abordado como una investigación sobre un individuo, grupo, organización, comunidad o sociedad visto y analizado como una entidad (Martínez, 2011, p. 23).
Desde esta perspectiva, y atendiendo a los procesos de triangulación que caracterizan al estudio de caso en el que se debe priorizar fuentes de datos y diferentes herramientas como: documentos, archivos, entrevistas, observación, entre otros (Stake, 2005; Yin, 2003), para que en cumplimiento de su sentido epistemológico se categorice, explique y comprenda el objeto investigado (Hernández, et al, 2014, p. 164); en esta oportunidad, el estudio de las características de los procesos curriculares desarrollados por el docente de ciencias naturales de la Institución Educativa Los Venados del municipio de Cereté, que constituyen el caso objeto de estudio de la presente investigación.

Las fases de investigación desarrolladas en el estudio y representadas de forma sistémica en la tabla 1, corresponden a tres momentos: un primer momento de análisis conceptual de la estructura curricular propuesta en la Institución Educativa objeto de indagación, seguido de un segundo momento sobre la interpretación del ejercicio docente mediante una entrevista semiestructurada, cuyo análisis está correspondido con el uso del software Atlas-ti. Finalmente, un tercer momento en la deconstrucción en el que se realiza un análisis documental al plan curricular de ciencias naturales del grado objeto de estudio, desde el enfoque de integralidad. 
Tabla 1. Sistema de categorías-Diseño metodológico.

\begin{tabular}{|c|c|c|c|c|c|}
\hline Objetivo & Categoría & Subcategorías & Momentos & Técnicas & Instrumentos \\
\hline $\begin{array}{l}\text { Interpretar } \\
\text { los procesos }\end{array}$ & & $\begin{array}{l}\text { Estructura } \\
\text { curricular }\end{array}$ & 1 & $\begin{array}{l}\text { Análisis } \\
\text { conceptual }\end{array}$ & $\begin{array}{c}\text { Guía de } \\
\text { observación }\end{array}$ \\
\hline $\begin{array}{l}\text { curriculares } \\
\text { a partir de la } \\
\text { deconstrucción } \\
\text { de la enseñanza }\end{array}$ & & Ejercicio docente & 2 & Entrevista & $\begin{array}{l}\text { Guía de } \\
\text { Entrevista } \\
\text { semi- } \\
\text { estructurada }\end{array}$ \\
\hline $\begin{array}{l}\text { de las ciencias } \\
\text { naturales desde } \\
\text { un análisis } \\
\text { hermenéutico } \\
\text { de la estructura } \\
\text { curricular, del } \\
\text { ejercicio de } \\
\text { planeación } \\
\text { docente y del } \\
\text { plan curricular. }\end{array}$ & $\begin{array}{c}\text { Integralidad } \\
\text { Curricular }\end{array}$ & Plan curricular & 3 & $\begin{array}{c}\text { Análisis } \\
\text { documental }\end{array}$ & $\begin{array}{c}\text { Matriz de } \\
\text { análisis }\end{array}$ \\
\hline
\end{tabular}

\section{RESULTADOS}

\section{Análisis conceptual de la estructura curricular propuesta en la Institución Educativa.}

El punto de referencia que se toma para el plan de estudios analizado, es el componente normativo expresado en la Ley General de Educación Colombiana, de febrero 8 de 1994, los lineamientos curriculares, estándares básicos de competencias y derechos básicos de aprendizaje. En la Ley General el artículo 5 que define los fines de la Educación colombiana, se puede especificar la caracterización epistemológica, para la enseñanza de las ciencias, el artículo 11 expresa la distribución por niveles: preescolar, básica y media.

Se plasma en los lineamientos la estructura curricular de las Ciencias Naturales por niveles así:

- $\quad$ Nivel medio: Grado $10^{\circ}$ y $11^{\circ}$. Entorno vivo y entorno físico - químico, profundización en química y física.
- Nivel básico: Grados del $1^{\circ}$ al $9^{\circ}$. Entorno vivo, entorno físico, entorno químico y entorno ambiental.

Como se aprecia según lo establecido en la misma ley hasta el noveno $\left(9^{\circ}\right)$ grado, una propuesta curricular debe ser orientada por procesos que necesariamente revisten integralidad desde lo físico, lo químico, lo biológico y lo ambiental, y que permite comprender el mundo natural desde sus relaciones sistémicas.

En los lineamientos curriculares se encuentran aspectos conceptuales que pueden generar cambios de actitud profesional ante la comprensión de los fenómenos de las Ciencias Naturales, donde se busca estudiantes con el desarrollo del pensamiento científico, contar con una teoría integral del mundo natural, que permite una orientación para la toma de decisiones.

Un análisis detallado de la estructura curricular expuesta en el plan de estudios para el caso particular que convoca la presente investigación, conduce al análisis global de la propuesta 
curricular presente desde lo general a lo específico, en este caso el grado $7^{\circ}$, ya que la propuesta así lo determina, presentándose una estructura curricular distribuida por grados como se muestra en la tabla 2.

Tabla 2. Distribución de las asignaturas de ciencias naturales.

\begin{tabular}{|c|c|c|c|}
\hline Asignatura & Grado & Nivel & Intensidad Horaria Semanal \\
\hline Ciencias Naturales & $1^{\circ}$ a $9^{\circ}$ & básica primaria y secundaria & 4 \\
\hline \multirow{2}{*}{ Biología } & $6^{\circ}$ y $7^{\circ}$ & básica secundaria & $\begin{array}{c}4 \text { (las correspondientes a } \\
\text { ciencias naturales) }\end{array}$ \\
\hline Química & $6^{\circ}$ y $7^{\circ}$ & básica secundaria & 1 \\
\hline \multirow{2}{*}{ Física } & $6^{\circ}$ y $7^{\circ}$ & No aplica & No aplica \\
\cline { 2 - 4 } & $10^{\circ}$ y $11^{\circ}$ & Media & 2 \\
\hline
\end{tabular}

Como se observa, esta distribución académica es disciplinar y muy significativa, ya que se propone a las Ciencias Naturales como asignatura independiente, e incluso asilada del mismo concepto de Ciencias Naturales, y mucho más la división de biología, química y física, totalmente disgregados y con una particularidad, que a los estudiantes de básica secundaria $\left(6^{\circ}\right.$ a $\left.9^{\circ}\right)$, no se les brinda la oportunidad de estudio de las Ciencias Físicas. Lo que indica de alguna forma, ausencia de integralidad curricular y en su lugar el asignaturismo segmentario y disgregado, que se aleja de un marco interdisciplinar importante de acuerdo a la normatividad colombiana y que moviliza el proceso de enseñanza de las Ciencias Naturales, cuya integralidad debe responder a una continuidad lógica de visión sistemática y holística que articule todos los sistemas vivos (Bertafalanffy, 1981; Cepra ,2000).

\section{ANÁLISIS DEL EJERCICIO DE PLANEACIÓN DOCENTE.}

Partiendo de los datos recabados tras la implementación de una entrevista semiestructurada con el docente del caso y atendiendo directamente al proceso de deconstrucción desde su propio ejercicio, el análisis hermenéutico y a profundidad de las respuestas brindadas por el docente y bajo la óptica del análisis de contenido con ayuda del software para organización y tratamiento de datos cualitativos (Atlas.ti); tras procesos internos de codificación abierta, selectiva y axial, permite la caracterización del docente participante al respecto de su ejercicio en relación con la integralidad curricular (Figura 1).

Así las cosas, se genera a continuación una interpretación del fenómeno con direccionamiento a la deconstrucción como parte de generalizaciones extraídas de casos de investigación-acción pedagógica realizados entre 1998 y 2004, que apoyan la tesis de la construcción de saber pedagógico por parte de los docentes, en procesos de deconstrucción, reconstrucción y evaluación de la práctica, generando entonces; un proceso de crítica social al develar los problemas de la práctica particular, pretendiéndose una transformación de la práctica pedagógica durante el desarrollo mismo de la investigación educativa, de manera que, llevándose la investigación al aula, trazándose de manera sistemática y rigurosa, se constituye un proceso de investigación sobre la práctica en el laboratorio de las aulas (Restrepo, 2006). 


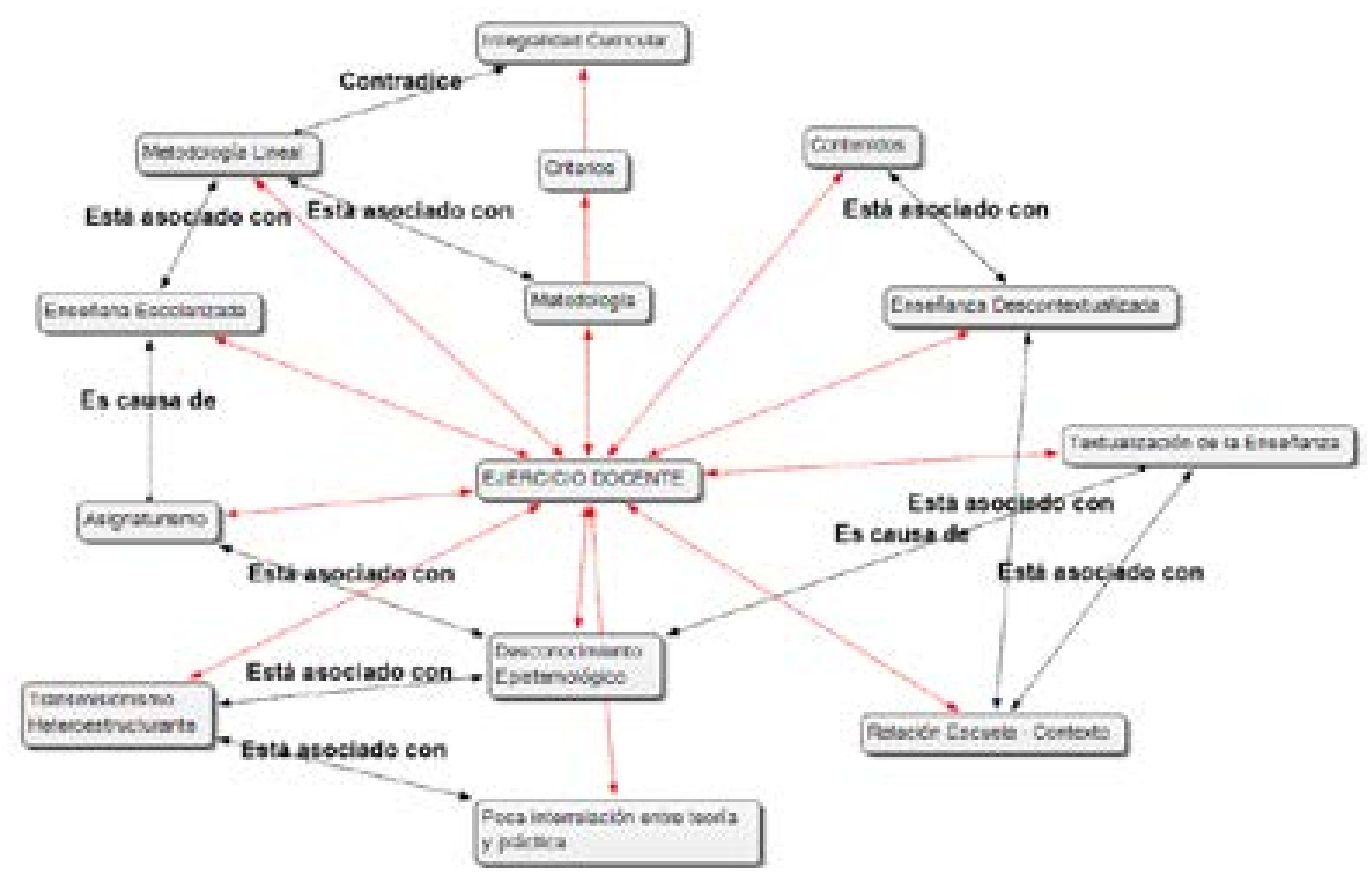

Figura 1. Red semántica de la categoría "Ejercicio Docente". Fuente: elaboración propia.

El análisis permite sustentar que el docente en su ejercicio profesional no aplica un pensamiento total para enseñar ciencias naturales, se demuestra la ausencia de un currículo con características de integralidad, por lo que se puede deducir que el actuar docente está sumergido en el campo eminentemente disciplinar, independiente, especificado por asignaturas: Biología, Química y con la ausencia del pensamiento Físico. Además, se resalta que la distribución académica se aplica por conveniencia de los profesores, y el criterio es por su campo disciplinar, por eso existen los profesores de Física, Química, Biología y no de Ciencias Naturales.

En consonancia con lo anterior, un acercamiento más específico respecto a la práctica docente para comprender su complejidad desde los docentes en su quehacer, permite desde sus percepciones (entrevista) deconstruirla. En efecto:

"No estoy trabajando una parte integral por área ya que trabajamos por asignatura esta la trabajamos por los lineamientos curriculares y estándares básicos de competencia DBA trabajamos una matriz de desempeño" (Entrevista 1).

Ante la respuesta dada por el caso no existe ninguna interrelación disciplinar desechando cualquier característica que implique un currículo integrado. Por lo tanto, se presentan falencias en la comprensión de la naturaleza de las ciencias, que se aborda desde lo epistemológico, histórico y social sin tener presupuesto en éstas prácticas curriculares, generando concepciones lineales del mundo circundante y su limitada comprensión.

Es determinante resaltar en el docente la carencia de criterios claros y básicos que orienten la selección y organización de contenidos. Ante este hallazgo, resulta importante destacar que en estos momentos históricos de evolución constante de la pedagogía y concepciones curriculares, se plantee la necesidad de seleccionar y organizar contenidos articulados a las realidades contextuales que respondan a las características del mismo contexto y de sus estudiantes, y que a su vez les den 
significados que se traduzca en formas de pensar, sentir, actuar y valorar frente a los problemas que se presentan constantemente en la vida natural, social y cultural, para darles respuesta satisfactoriamente. De tal modo que se corresponda con lo expuesto por García (1995), al concebir un currículo integral donde el quehacer docente esté bajo un prisma epistemológico diferente, en concordancia con una metodología opuesta a la reduccionista y parcelaria, para concebir el análisis con una visión global y generalizada.

En este orden de ideas y profundizando en las respuestas del docente, ante la expresión:

"Me baso en la secuencia de los textos y hay una jerarquía que se ven en los DBA con su cierta autonomía" (Entrevista 1).

Se ve manifiesto que no hay una interrelación entre la enseñanza de las ciencias y el contexto social; como lo manifiesta el docente son acciones esporádicas, que no llegan a tener significación en la vida de los estudiantes, son simples momentos estratégicos. Se sabe que es determinante que para enseñar y aprender ciencias debe existir una relación constante y permanente con lo contextual, lo cultural y lo social, pues es de conocimiento que las ciencias son un proceso de construcción social, permitiendo al estudiante resolver situaciones problémicas desde sus propias realidades, y por ende generando altos grados de motivación. En consonancia, los docentes deben ser conocedores profundos del saber disciplinar para llegar a entender los vínculos con las demás áreas del conocimiento y puedan enseñar las disciplinas desde esa perspectiva de integración (Beane, 2005).

Lo anterior es consecuencia del trabajo metodológico que aplica el docente, para quien es común recitar el contenido del texto y el estudiante en su rol de aprendiz que toma apuntes sobre lo que expresa el docente directamente del texto, es decir, transcribir los enunciados del texto a la libreta del estudiante sin ningún tipo de filtro, ya que el texto por lógica está mejor, a lo que el investigador denomina: "crimen pedagógico". Así las cosas, es frecuente lo que se desarrolla en el aula de clases sobre la transmisión lineal del conocimiento con un pensamiento hetero-estructurante y siguiendo en su discurso lo que dice el texto, el estudiante con una actividad receptivo- pasiva, escribiendo lo que ordena el profesor sin ninguna oportunidad de análisis; el recurso didáctico es repetir lo que manifiesta el docente, desarrollando la clonación de contenidos y memorística como fuentes de saber.

Por su parte, en correspondencia con lo expresado por el docente:

"Le repito, no estamos trabajando la integralidad. Sin embargo, tomo en la mayoría de los casos relaciones de su cultura para llevar algunos casos específicos a temas específicos" (Entrevista 1).

Se puede observar que no existen esquemas metodológicos múltiples que le permita a los estudiantes apropiarse de los saberes desde sus mismas realidades, con las esporádicas salidas, y la utilización de videos como lo manifiesta el docente; no presenta un proceso epistémicometodológico consistente y no garantiza la adquisición de saberes, más aún cuando la especificidad de las ciencias naturales recurre a hechos experimentales y se deben abrir espacios desde el mismo contexto, donde el estudiante tenga la oportunidad de demostrar y reconstruir.

Asimismo, concibiéndose el aspecto metodológico, el participante expresa que:

"No trabajo de forma integral, sin embargo, utilizo recursos metodológicos como las salidas de campo en los contextos de la escuela, para observar algunos fenómenos o condiciones del medio natural para que ellos analicen y deduzcan 
teniendo en cuenta unas orientaciones (guías prediseñadas); proyección de videos, cuando se necesita mostrar o entrar en el mundo microscópico; desarrollo de talleres en clase." (Entrevista 1).

Ante la respuesta dada por el caso, se consolida la posición del investigador, donde se manifiesta, que los esquemas metodológicos aplicados en las ciencias naturales no pueden ser métodos únicos, es evidente aplicar metodologías múltiples, contextuales, experimentales desde su que-hacer, interrogando y dando solución a sus propias inquietudes, donde se aprende significativamente.

Los procesos metodológicos lineales permiten un aprendizaje lineal y descontextualizado sin acceder a la interrelación de los contenidos de las ciencias naturales con su vida cotidiana, el sistema estratégico es limitado sin aprovechar el contexto y brindar respuestas a sus múltiples interrogantes, se utiliza el conocimiento cotidiano esporádicamente, desaprovechando la riqueza del conocimiento natural, social y cultural; el desarrollo escolar es de aula, no se utiliza el medio natural para que los estudiantes interactúen directamente con la naturaleza, comprendan y brinden respuestas a los interrogantes que se les presenta continuamente.

Siguiendo el orden relacionado con el proceso de deconstrucción del quehacer docente de las ciencias naturales, el sujeto investigado, considera que:

"El proceso se está trabajando por asignatura Biología, química además, los docentes se seleccionan por perfil y es el caso que los docentes que algunos manifiestan no tienen la afinidad por la física, química o biología por eso siendo del mismo área hay docente de física, biología y química no se trabaja en ciencias naturales". (Entrevista 1).
De acuerdo con esto, se resalta que la distribución académica se aplica por conveniencia de los profesores, y el criterio es por su campo disciplinar, por eso existen los profesores de Física, Química, Biología y no de Ciencias Naturales. Por lo tanto, no existe ninguna interrelación disciplinar desechando cualquier característica que implique un currículo integrado.

Por otro lado, pero sin alejarnos del proceso de deconstrucción de la práctica pedagógica, la expresión:

"Tomo aspectos significativos de la cotidianidad partiendo de los preconceptos que los estudiantes tienen o poseen y los llevo al tema que se está trabajando para que se haga más dinámica y comprensible la clase" (Entrevista 1).

Muestra que no existe un esquema metodológico que le permita interrelacionar los contenidos de las ciencias naturales con la vida cotidiana de los estudiantes únicamente se utilizan los conocimientos cotidianos de los alumnos desaprovechando la riqueza del conocimiento natural.

La enseñanza tradicional de la ciencia es eminentemente escolástica de aula, no se aprovechan las realidades naturales donde conviven e interactúan los estudiantes, no se les permite explotar sus saberes y dar respuestas a los interrogantes desde su propio contexto, de manera que, se demuestra que no hay una interrelación entre la enseñanza de las ciencias y el contexto social, como lo manifiesta el docente. Se observan acciones esporádicas, que no llegan a tener significación en la vida de los estudiantes, son simples momentos estratégicos.

En esta misma línea de análisis y como se mencionó anteriormente, en relación con la expresión: 
"Utilizo en los procesos de clase las salidas a campos, proyección de video" (Entrevista 1).

Se detecta que el proceso enseñanza aprendizaje es lineal, unilateral, directo; por lo tanto el sistema estratégico es limitado sin aprovechar el contexto y dar respuestas a sus interrogantes. Cuando los esquemas para la enseñanza de las ciencias carecen de integralidad, se convierte en verbalista, libresca, textual y no se aprovecha las interacciones contextuales, las cuales deben ser experimentadas desde sus realidades, lo que se traduce en punto de motivación, por tratarse del camino más seguro hacia la respuesta de sus interrogantes.

Finalmente, en relación con el aspecto didáctico, el participante considera que:

"No se relaciona ya que el trabajo es fraccionado como dije anteriormente aprovechamos la vida natural, ambiental para partir de sus conocimientos previos" (Entrevista 1).

En efecto, se muestra que la enseñanza de la ciencia es fraccionada sin tener ningún ápice de integralidad. Es determinante que en los currículos para la enseñanza de las ciencias se realicen cambios estructurales que trasciendan en la vida del docente y en la vida del estudiante para cualificar permanentemente el proceso de enseñanza-aprendizaje de las ciencias naturales.

A manera de síntesis, a la luz de los procesos de deconstrucción de la práctica pedagógica realizada por el docente del área de las ciencias naturales, que es muy diciente respecto al hecho que los docentes de hoy no hayan trascendido a las enseñanza de las ciencias naturales confiriéndole oportunidad a la integralidad, esa manifestación de la enseñanza específica no permite que el estudiante comprenda el mundo como existe realmente, lo percibe como un mundo fragmentado desligado de su propia realidad, por ello existe la necesidad de cambiar los currículos rutinarios y descontextualizados por currículos integrados como lo mencionan Rodríguez-Learte, et al. (2018) y cuya implementación promueva el desarrollo de habilidades de aprendizaje en el que se construyan procesos de manera flexible e individualizada.

Ante este panorama, algunos docentes se conforman con utilizar los textos guías que no responden en lo mínimo a las propias realidades de sus estudiantes, es una forma de realizar procesos sin aplicación de acciones mentales, es la simple repetición, memorización de contenidos sin resolver situaciones de su vida cotidiana y por ende se mostrarían distantes a responder inquietudes e interrogantes que se les presenta, de manera que la percepción entre las relaciones currículo institucional, didáctica de las ciencias naturales y estándares por competencias no apuntan a una integralidad, ni el uso de un marco interdisciplinar donde el discente pueda tener un proceso formativo competente en el contexto en el que interacciona social y socio-cognitivamente atendiendo una perspectiva holística de su realidad.

\section{ANÁLISIS DEL PLAN CURRICULAR EN LA ENSEÑANZA DE LAS CIENCIAS NATURALES.}

En este momento de la deconstrucción curricular, se diseñó una matriz de análisis para examinar el plan curricular que acompaña el proceso de planeación docente a la luz de ejes como la intensidad horaria, componentes curriculares (estándares básicos de competencias y derechos básicos de aprendizajes), selección de contenidos, organización y secuencia de contenidos y el carácter disciplinar.

Como resultado de este proceso, se observa de manera general, que la distribución académica responde al asignaturismo, sin embargo, en el inciso 6, pág. 21, del, plan de estudio se plantea el objetivo con característica de integral, 
tanto en la enseñanza de las ciencias, como la formación del ser humano; en la página 22 inciso 7 , se propone el estudio por asignaturas específicamente, Biología y Química, no se tiene presente la Física y la Educación, se demuestra el estudio disciplinar, segmentado y descontextualizado.

En la malla curricular del grado séptimo $\left(7^{\mathrm{a}}\right)$, objeto de la investigación, se titula el estudio de Ciencias Naturales, los referentes que se toman son los estándares curriculares y los derechos básicos de aprendizaje establecidos desde el Ministerio de Educación, estos corresponden al estudio de las ciencias, que en esta propuesta se estudia por disciplinas, biología, química, educación ambiental y no el estudio de la física. La selección de contenidos a pesar que el documento sustenta una base epistemológica, sistémica, holística, formación científica básica, integración de procesos físicos, químicos, biológicos y ambientales, (plan de estudio, pág 56.); se hace en forma disciplinar y fragmentada, respondiendo al estudio biológico, químico y ambiental, es decir no hay criterios que respondan a intereses y necesidades de los educandos y a la resolución de problemas desde su contexto.

La secuenciación es una consecuencia de lo anterior, no está articulado ni a los estándares ni a las propias realidades de los estudiantes, son contenidos textualizados, que más responden a situaciones externas, que a la solución de problemas de la vida cotidiana en el contexto particular de estudio. En general no responden a un discernimiento de las propias realidades de la institución y del contexto por eso son contenidos fragmentados y descontextualizados.

Se puede deducir, que no hay claridad con el estudio de las ciencias naturales, a pesar de que se propone su estudio para la educación básica; se pudo comprobar que se realiza por asignaturas, biología y química, totalmente independientes, fragmentadas $y$ descontextualizadas, con la dificultad de no estudiar la física en el grado séptimo, privando a los educandos de un componente natural básico para comprender la complejidad de la naturaleza, y la educación ambiental reducida a un solo período académico; es un reduccionismo que no le permite al estudiante desarrollar un pensamiento integral y complejo, y mucho menos comprender e interactuar en su propio mundo.

Por otra parte aterrizando en la población específica objeto de indagación como se mencionó en líneas anteriores, correspondiente al grado séptimo, la planeación curricular es desarrollada utilizando una matriz, distribuida en esquemas donde especifica estándares, derechos básicos de aprendizaje, contenidos y evidencias por períodos académicos. Asimismo, los estándares y derechos básicos son textuales de la norma ministerial y aparecen en los cuatro periodos académicos. El derecho básico en su defecto, se refiere a las cadenas tróficas ciclos energéticos (Plan de estudio, pág. 54, 55 ,56), los contenidos que deben apropiarse los estudiantes se refieren a procesos biológicos, fotosíntesis, nutrición, digestión circulación respiración, sin ninguna lógica con los estándares y los derechos básicos (Ver Figura 2).

En concordancia con la línea del análisis de contenido curricular, se destaca que el estudio de la química, se realiza en forma disciplinar independiente del estudio de los demás componentes de las ciencias; lo ambiental se plantea para su estudio en su periodo académico, totalmente aislado, fragmentado como contenido independiente. La física no se estudia en la básica secundaria, los estudiantes desconocen este componente académico tan necesario para poder comprender los conceptos básicos de la ciencia. En definitiva, se observa un proceso físico alejado de los procesos químicos, bilógicos y ambientales. 
La entrevista al docente que develó especificidades para el segundo momento de la deconstrucción, también proporcionó luces para el análisis del plan curricular y dichos rasgos surgieron en el análisis hermenéutico como aporte a la categoría denominada para este estudio como "Plan curricular", cuyo proceso de codificación, condujo a la red semántica que se muestra a continuación:

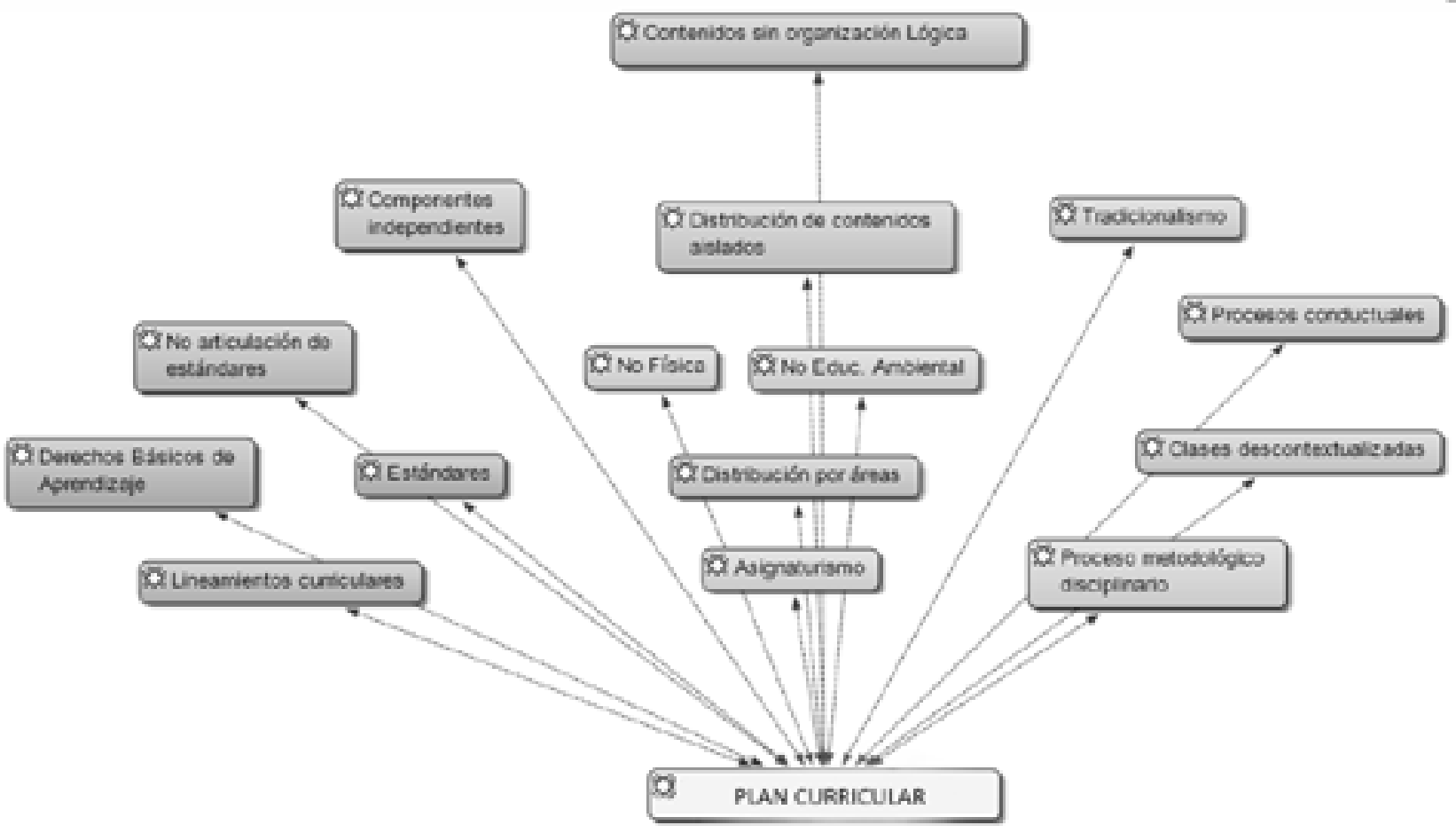

Figura 2. Red semántica de la categoría "Plan Curricular". Fuente: elaboración propia.

En correspondencia con lo anterior, y en coherencia con los postulados expuestos en las teorías relacionadas a la interdisciplinariedad y la epistemología docente de las Ciencias Naturales, se debe dar claridad en que, el objetivo de la ciencia es comprender la realidad y con las características actuales y la multiplicidad de disciplinas en donde un mismo objeto puede ser estudiado por muchas ciencias.

En consecuencia, se debe entender la necesidad de organización o direccionamiento de los contenidos partiendo de conceptos sistémicos y no por unidades temáticas individuales, asociado a que el mundo es global y que todo está relacionado. Las diferentes dimensiones que constituyen las sociedades son interdependientes. Como afirma Torres Jurgo (1998) "El currículo globalizado e interdisciplinario se convierte en una categoría de paragua capaz de agrupar una variedad de prácticas educativas que se desarrollan en las aulas y es un ejemplo significativo de interés para mejorar los procesos de enseñanza aprendizaje".

La realidad natural por su origen, es compleja, todo está integrado por lo tanto las ciencias de la naturaleza así lo son; el docente debe interactuar ante estos procesos holísticos de la ciencia natural y el currículo por medio del 
concepto de "mundo de vida" (Habermas 1998; Lozano. O, y Villanueve O, 2016).

En esencia, consiste en generar un cambio paradigmático, en la visión integral de las ciencias naturales, que generen nuevas ideas y habilidades que se interioricen y se transfieran con las experiencias previas, contextos significativos e ideas integrales y no cuando se enseña partes abstractas y fragmentadas (Beane, 2005).

El contexto es complejo de donde emergen constantes cambios, que trasciende en los problemas educativos, donde el docente debe articular esos cambios en su actuar, generando aprendizajes más flexibles, abiertos asociados a la realidad contextual y la vida cotidiana del estudiante (Lapasta, L. 2018). En consecuencia, docentes conocedores profundos del saber disciplinar y con capacidades para integrar saberes, y puedan enseñar las disciplinas desde esa perspectiva.

\section{CONCLUSIONES}

El proceso investigativo a través del análisis aplicado en la Institución Educativa Cristóbal Colón del corregimiento Los Venados Campanito, Municipio de Cerete, desde procesos de deconstrucción ha permitido reflexionar, que las decisiones curriculares se enmarcan en la disciplina y responsabilizan a docentes de acuerdo al perfil de estudio, razón por la que estos se identifican como de química, de física, de biología, su desarrollo es totalmente independiente; la aplicación es por asignaturas en un currículo descontextualizado, en donde las disciplinas se desarrollan por temáticas fragmentadas, sin tener presente la lógica del conocimiento; en donde no hay criterios claros que direccionen la organización y la selección de contenidos, esto se hace a partir de los textos guías y siguiendo parámetros ministeriales, que están previamente seleccionados desde otros contextos, además contenidos tan significativos como los de la física, se les niega a los estudiantes de educación básica secundaria, cuando hoy es importante el estudio de las ciencias desde las tempranas edades (Daza, S, \& Quintanilla, M, 2011).

Un aspecto a resaltar es que la selección de contenidos no tiene articulación con la realidad contextual de los estudiantes, se utiliza su contexto en el proceso enseñanza y aprendizaje como recurso para que los estudiantes observen aspectos de la naturaleza sin ninguna apropiación científica; la aplicación metodológica se enmarca en pedagogías tradicionales, con la aplicación de métodos transmisión-recepción, de mucha caracterización en nuestras instituciones educativas, en donde se manifiesta el memorismo y la pasividad del estudiante y el uso reiterativo del aula de clase, sin ninguna experimentación.

Otras consideraciones deconstructivas, a la luz de la revisión documental, conducen a comprender que, de manera general la propuesta curricular para la enseñanza de las ciencias no tiene sustento teórico entre lo se plantea en el plan de estudio y con lo que el estudiante aprende, y no existe lógica en la organización curricular de la estructura de las ciencias. Asimismo los estándares no especifican lo que se debe aprender en el grado séptimo por lo que la selección de contenidos no tiene criterios claros, respondiendo sólo a situaciones textuales, no a necesidades e intereses de los estudiantes que le permitan resolver problemas de su vida cotidiana.

Por su parte, los fundamentos son teóricos, no hay interacción entre la teoría y la práctica; clases de aula desconociendo la importancia del contexto como laboratorio para resolver problemas integrales de la ciencia; son modelos de enseñanza enmarcados en el tradicionalismo, procesos conductuales, memorístico y descontextualizados. Por consiguiente, es determinante apropiarse una metodología 
basada en un currículo integrado, que conlleve el desarrollo de un pensamiento integral, complejo, crítico, reflexivo, emancipador, en la aplicación de las ciencias naturales como un todo, asociado a la complejidad del mundo, donde interactuamos y brindar solución a los interrogantes que constantemente se presentan.

Ahora bien, ante los procesos de deconstrucción realizados, se pone de manifiesto la necesidad de una propuesta curricular basada en la integralidad, donde se contribuya a la construcción en la enseñanza de las ciencias naturales, articulados con las realidades contextuales de los estudiantes, que le permitan resolver situaciones desde su propia vida cotidiana. En este sentido, es pertinente un cambio de paradigma para enseñar ciencias, con base en la pedagogía integradora e interdisciplinar, generando procesos integrales, contextualizados, articulados a las propias realidades de los estudiantes. Una estructura curricular que responda al desarrollo de las ciencias, no así, a los simples conocimientos estandarizados, y descontextualizados.

\section{REFERENCIAS BIBLIOGRÁFICAS}

Aguilar-Barreto, A.J., Bermúdez-Pirela, V. y Hernández Peña, Y.K. (Ed.) (2018). Educación, cultura y sociedad: oportunidades para la investigación. Cúcuta, Colombia: Ediciones Universidad Simón Bolívar.

Beane, JA (2005). La integración del Currículum. Ed Morata.

Bertalanffy, L y Cols. (1981). Tendencia de LA. Teoría General de Sistemas Madrid.

Daza, S., \& Quintanilla, M. (2011). La enseñanza de las ciencias en las primeras edades. Su contribución a la promoción de competencias de pensamiento científico. Vol, 5 .

García, J.E. (1995): Epistemología de la complejidad y enseñanza de la ecología.
El concepto de ecosistema en la educación secundaria. Tesis doctoral inédita. Universidad de Sevilla

Habermas, J. (1988). Teoría de la acción comunicativa (Tomos I y II). Madrid: Taurus.

Hernández Peña, Y.K., Hernández, A., Navarro, M., Acevedo, L., Barrera, L. y Rozo, O. (2018). Deconstrucción y transformación de las prácticas pedagógicas en torno a la formación por competencias. En A.J. Aguilar-Barreto; V. Bermúdez Pirela y Y.K. Hernández Peña. (Ed.), Educación, cultura y sociedad: oportunidades para la investigación. (pp. 119-136). Cúcuta, Colombia: Ediciones Universidad Simón Bolívar.

Hernández, R., Fernandez, C., \& Baptista, P. (2014). Desarrollo de la perspectiva teórica: revisión de la literatura y construcción del marco teórico. Metodología de la Investigación ( $6^{\mathrm{a}}$ ed., pp. 58-87). México: McGraw-Hill.

Jelves Zárate, V. R. (2017). Develando el Currículo Integrado. Representaciones Sociales que en relación al Currículo Integrado habitan en profesores y profesoras de una escuela que practica la integración curricular. Paulo Freire, 17, 197. https://doi.org/10.25074/07195532.17.542

Lapasta, L. G. (2018). Experiencias múltiples de apropiación del conocimiento para la construcción de la práctica profesional docente en la formación de profesores universitarios de Ciencias Exactas y Naturales. INTERNATIONAL JOURNAL EDUCATION AND TEACHING (PDVL) ISSN 2595-2498, 1(1), 110-122.

Ley General de la Educación de 1994 y la Misión de Ciencia, Educación y Desarrollo de 1995.

López Rupérez, E. (1990).

Epistemología y didáctica de las ciencias. Un análisis de segundo orden. Enseñanza de las Ciencias, 8(1), pp. 65-74. 
Lozano, C. y Villanueva, G. (2016). Ciencia, Currículum y profesores: las Ciencias Naturales en la Educación Secundaria. Volumen 16 Número 1, pp. 1 - 28. DOI: http://dx.doi.org/10.15517/aie. v16i1.21714

Marín, F. (2012). Investigación científica. Una visión integrada e interdisciplinaria. Venezuela: Ediciones del Vicerrectorado Académico de la Universidad del Zulia.

Marín, G., Paredes, C., e Inciarte, G. (2017). Didáctica interdisciplinaria de las ciencias: aportes a su comprensión desde la aplicación de mapas lógicos de relaciones conceptuales. En Pirela, M., Almarza, F., y Caldera, C. (coord) Didácticas para el desarrollo del pensamiento interdisciplinar. REDPI (Red para el Desarrollo del Pensamiento Interdisciplinar). Universidad del Zulia, República Bolivariana de Venezuela. Maracaibo

Martínez, J. (2011). Métodos de investigación cualitativa. Silogismos de investigación, 8(1), 1-43.

Monje, C. (2011). Metodología de la investigación cuantitativa y cualitativa. Guía didáctica. Neiva, Colombia: Universidad Surcolombiana.

Morín, E. (2003) Articular las disciplinas: la antigua y la nueva transdisciplinariedad, Itinerario Educativo, No. 39-40, 189-205.

Munarriz, B. (1992). Metodología educativa I. Xornadas de Metodoloxía de Investigación Educativa (A Coruña, 23-24 abril 1991), coordinadores Eduardo Abalde Paz, Jesús Miguel Muñoz Cantero. A Coruña: Universidade da Coruña, Servizo de Publicacions, p. 101-116. ISBN: 84-600-8006-4

Pinto Contreras, R. (2008). El currículo crítico. Una pedagogía transformativa para la educación latinoamericana. Santiago, Chile: Editorial UC.
Restrepo, B. (2006). La Investigación-Acción Pedagógica, variante de la Investigación-Acción Educativa que se viene validando en Colombia. Universidad de Antioquia. P. 96

Rodríguez-Learte, A., González-Soltero, R., Rodríguez-Martín, I., Tutor, A. S., Sánchez, A. M., \& Gal, B. (2018). Liderando el cambio: hacia un currículo integrado para ciencias biomédicas. Experiencia de la Universidad Europea de Madrid. Revista de La Fundación Educación Médica, 21(4), 215. https://doi.org/10.33588/ fem.214.958.

Salgado Lévano, A. C. (2007). Investigación cualitativa: diseños, evaluación del rigor metodológico y retos. Liberabit, 13(13), 71-78.

Schunk, D. (2012). Teorías del aprendizaje: Una perspectiva educativa. México: Pearson.

Stake, R. (2005). Qualitative case studies. En N. Denzin \& Y. Lincoln (2005). The Sage handbook of qualitative research. Third edition. London: Sage Publications.

Torres Santome Jurjo. (1998). Las razones del currículo integrado" en globaliza e interdisciplinariedad integrado el currículo. Madrid, Morata.

Valbuena, E. (2007). El Conocimiento Didáctico del Contenido Biológico. Estudio de las concepciones disciplinares y didácticas de futuros docentes de la Universidad Pedagógica Nacional (Colombia). Tesis doctoral. Universidad Complutense de Madrid (ISBN 978-84-6693101-4).

Vivas, S. L., Mancipe, H. D. y Aguilar-Barreto, A. J. (2017). Caracterización de las prácticas pedagógicas de lengua castellana en educación básica secundaria. En J. Gómez Vahos., A.J. Aguilar-Barreto., S.S. Jaimes Mora., C. Ramírez Martínez., Hernández Albarracín., J.P. Salazar Torres., J. C. Contreras Velásquez., y J.F. Espinosa Castro (Eds.). Prácticas pedagógicas. 
(pp. 1219-1252) Maracaibo, Venezuela.

Ediciones Universidad del Zulia.

Yin, R. (2003). Case study research.

Design and methods. US A: Sage Publications 